\title{
Student Physical Fitness Test System and Test Data Analysis System Based on Computer Vision
}

\author{
Ling Wang ${ }^{1}$ and Sitong Chen $\mathbb{D}^{2}$ \\ ${ }^{1}$ School of Physical Education, Shenyang University, Shenyang, 110044 Liaoning, China \\ ${ }^{2}$ School of Mechanical Engineering and Automation, Northeastern University, Shenyang, 110819 Liaoning, China \\ Correspondence should be addressed to Sitong Chen; 1510112@stu.neu.edu.cn
}

Received 13 January 2021; Revised 19 February 2021; Accepted 10 March 2021; Published 17 May 2021

Academic Editor: Wenqing Wu

Copyright ( 2021 Ling Wang and Sitong Chen. This is an open access article distributed under the Creative Commons Attribution License, which permits unrestricted use, distribution, and reproduction in any medium, provided the original work is properly cited.

\begin{abstract}
Computer vision technology is one of the main research directions of artificial intelligence. With the rapid growth of image or video data scale and the improvement of computing power, computer vision technology has achieved unprecedented development in recent years and is widely used in a variety of scenes. This study mainly discusses the design of student physical fitness test system and test data analysis system based on computer vision. This study is mainly based on the motion attitude determination algorithm to identify the motion. In hardware configuration, the key is CPU and GPU. The model realizes large-scale matrix computation based on the parallel computing power provided by GPU and uses CPU to realize data reading and preprocessing. The assessment controller is responsible for the transmission of instructions and status information and controls the operation of the entire pitch assessment system. It is the control center of the entire system. ZigBee wireless communication technology is adopted as the communication method of human posture measurement terminal and assessment controller. The input image is preprocessed through scaling and standardization. The image is scaled to the resolution of $224 \times 224$ when input, which is performed to realize data parallel training. The image was changed by means of random horizontal flip, random rotation, and color change to achieve the effect of expanding the dataset. Then, the test evaluation module was used to evaluate various test indexes of the body. During the sit-up test, nine out of 10 sit-ups can be accurately counted and the recognition rate reaches 90 percent. The results show that the system designed in this study has high accuracy and good performance, which can be used for the physical fitness test and test data analysis of students.
\end{abstract}

\section{Introduction}

In the early days, due to the backwardness of the economy, our people's physical requirements only stayed on the basis of food and clothing. Later, with the development of the economy and the improvement of living standards, people paid more and more attention to the improvement of their overall physical fitness. With the improvement of the quality of life of our people and since the reform and opening up, China has strengthened the communication with the United States and many other countries in the aspects of economy, culture, science and technology, and political system and directly introduced many foreign advanced ideas and practices in the aspect of physical improvement.
Our country has gradually launched a physical fitness test program combined with physical examination during the compulsory education stage. So far, basic schools in our country have organized students to conduct physical examinations in school hospitals and physical fitness tests in sports venues. The health of students has been regarded as an important guarantee for the country's new force. In recent years, the term "health records" has become popular in my country's medical circles. Although many difficulties have been encountered in implementation, now basically only large hospitals have established electronic medical records instead of national unified health records. As for physical fitness testing, information technology has not been fully promoted. Only independent systems equipped with physical fitness testing equipment cannot comprehensively reflect 
health conditions. Due to the lack of information technology and integrated system for physical fitness testing in China, this has brought great challenges to the research of this subject [1].

Computer vision methods have been applied to cell segmentation and feature extraction, while machine learning methods have been developed to help phenotype classification and clustering of data obtained from biological images [2]. Grys et al. believe that with the latest developments in high-throughput automated microscopes, there is an increasing demand for effective computing strategies for analyzing large-scale image-based data. Here, they outline the common computer vision and machine learning methods used to generate and classify phenotypic profiles, focusing on the general biological uses of each method. Their research lacks data [3]. Yeung et al. introduced VideoSET, which is a method for evaluating video summaries through text. It can evaluate the extent to which the video summary can retain the semantic information contained in the original video [4]. They observed that semantics are most easily expressed in words and developed a text-based evaluation method. Their research has no practical significance [5]. Chang proposed a pixel-based cost calculation method that uses weighted distance information for cross-scale stereo matching. The method he proposed uses a hierarchical structure to accurately estimate the disparity value in a uniform area. He also uses distance information to supplement the pixel-based cost function. His research sample data is insufficient [6]. Zaki introduced a novel method for binary classification of twowheeled road users at dense mixed traffic intersections. Classification is a monitoring procedure used to distinguish electric and nonelectric (manpowered) bicycles. First, use object recognition methods to detect and track road users. Then, select classification features from the collected trajectories. Functions include maximum speed, rhythm frequency, and acceleration-based parameters. Experiments were conducted on a video dataset from Shanghai, China, where cyclists and motorcycles tend to share major road facilities. A sensitivity analysis was performed to evaluate the quality of selected features to improve the accuracy of classification. His research is not novel enough [7]. Meireles proposes an automatic and direct method to classify tooth wear in the oral cavity. The eight extracted teeth were etched with acid for different times to produce abrasion and scanned with an intraoral optical scanner. Computer vision algorithms are used for alignment and comparison between models. He estimated the amount of wear and determined the reliability through visual scoring. His research has no practical significance [8]. Wang proposed an automatic flexible printed circuit (FPC) defect detection method based on computer vision, which provides a method for calculating dimensionality increment matrix and image segmentation combined with fuzzy clustering algorithm. The visibility of the segmented image and the segmentation accuracy of the defective image are guaranteed. The method he proposed is not accurate [9].

This research mainly discusses the design of student physical test system and test data analysis system based on computer vision. This research is mainly based on the motion recognition algorithm for motion recognition. In the hardware configuration, the key is CPU and GPU. The model realizes large-scale matrix calculation based on the parallel computing capability provided by GPU and uses $\mathrm{CPU}$ to realize data reading and preprocessing. The assessment controller is responsible for the transmission of instructions and status information and controls the operation of the entire pitch assessment system. It is the center of the entire system control. ZigBee wireless communication technology is used as the communication method between the human body posture measurement terminal and the assessment controller [10]. The input image is subject to preprocessing steps of scaling and standardization. The image is scaled to a resolution of $224 \times 224$ during input. This step is performed to achieve data parallel training. Three methods of random horizontal flip, random rotation, and color change are used to change the image to achieve the effect of expanding the dataset, and then, the test evaluation module is used to evaluate various test indicators of the body.

\section{Student Physical Fitness Test System}

2.1. Computer Vision. In recent years, due to the wide application of computer vision in intelligent surveillance and surveillance, health and medicine, sports and entertainment, robotics, drones, and driverless cars, it has become more and more important and effective [11-13]. Depth information is one of the most important elements for generating three-dimensional (3D) content [14]. The stereo matching method uses binocular characteristics to estimate depth information $[15,16]$. The estimated depth information is usually represented by the disparity value [17]. Therefore, two slightly different viewpoints are used to find the disparity value. However, in a uniform area, since the area is untextured, the corresponding point finding is problematic [18].

Many computer vision and imaging problems are learned from large-scale datasets, which have millions of observations and features. A novel effective learning scheme tightens the sparsity constraint by gradually deleting variables based on criteria and schedules $[19,20]$. The fascinating fact that the size of the problem continues to decrease throughout the iteration process makes it particularly suitable for big data learning [21], generally applicable to the optimization of any differential loss function and find applications in regression, classification, and ranking. The resulting algorithm incorporates variable screening into the estimation and is very simple to implement [22], providing theoretical guarantees of convergence and selection consistency. In addition, one-dimensional piecewise linear response functions are used to solve nonlinear problems, and second-order priors are applied to these functions to avoid overfitting $[23,24]$.

2.2. Motion Attitude Determination Algorithm. This algorithm does not record all the angle values and then analyzes and processes, but uses a mobile real-time determination method to obtain the effective number of cycles, collect and record key information, and use a possible cycle as the 
algorithm execution cycle to extract the effective cycle [14]. The core idea of the algorithm is to take the maximum value of a certain number of accumulated records as the peak and the minimum value of the accumulated record of a certain number of times as the trough. If it is greater than the set evaluation standard, it will be recorded as a valid period [5, 16]. The execution cycle of the algorithm is the execution cycle of the entire program; each time the current angle value is obtained as an input variable for judgment [25].

The algorithm can be obviously divided into three parts. The first part is to judge the angle accumulation and accumulation. Each time it is executed, the current value and the recorded maximum and minimum values are used for judgment; the second part is to identify the number of accumulation and subtraction. The peaks and troughs are accumulated to 20 times, and it is considered that there is a trough. Similarly, there is a trough after the accumulation of 20 times. The identification of the peak is a condition for trough identification. The identification process of the two is similar; the third part is after obtaining the peak and peak value. To determine whether it is a qualified period, use a pair of recorded peak and valley values as the key variable to accurately obtain the possible effective period. In actual applications, time judgment is added, that is, the limit time for the human body to complete a pitching action is $300 \mathrm{~ms}$. If the cycle is lower than this time, it is considered to be an abnormal fluctuation and is not adopted $[18,26]$. The execution stage refers to the process in which the neural network processes the input information and generates the corresponding output [19]. In the execution stage, the connection structure and weight coefficients of the network have been determined and will not change. At this time

$$
U(t+1)=\sum_{j=1}^{n} W_{i j}(t+1) X_{j}(t)-\theta(t+1) .
$$

Among them, $W_{i j}$ is the weight coefficient of the connection between the $i$ neuron and the former $j$ level neuron. The motion attitude determination optimization algorithm has faster convergence speed and better optimization effect, which is very suitable for large-scale data or large-scale model problems.

$$
\begin{aligned}
m_{t} & =\beta_{1} m_{t-1}+\left(1-\beta_{1}\right) g_{t}, \\
v_{t} & =\beta_{1} v_{t-1}+\left(1-\beta_{1}\right) g_{t}^{2}, \\
\theta_{t+1} & =\theta-\frac{\eta}{\sqrt{v+\varepsilon}} m .
\end{aligned}
$$

Among them, $m_{t}$ and $v_{t}$ calculate the first and second moments of the gradient [6].

2.3. Network Input Layer. For the BP network, the input layer acts as a buffer memory and can add data to the network. The number of nodes depends on the dimensionality of the data source. The output layer outputs the processing results of the network, and the number of nodes can be designed according to the requirements of use. For sports events, the physical status of students is affected by many factors; among which gender, age, height, weight, and the 14 physical fitness test indicators mentioned above have a greater impact on determining the physical status of students. We must evaluate a student's overall physical fitness status or individual physical fitness status in terms of running, jumping, and shooting based on these influencing factors. Taking the evaluation of students' comprehensive physical fitness status as an example, we set the dimension of the network input layer to 18 , and each node represents the student's gender, age, height, weight, and 14 physical fitness test indicators; in actual training, the current physical state of students is divided into three states: poor, normal, and good. Therefore, the dimension of the network output layer is set to 3, representing these three states, respectively.

\section{Student Physical Fitness Test System Experiment}

3.1. Experimental Environment. The operating system configuration is shown in Table 1. In the hardware configuration, the key is CPU and GPU. Although the model is based on the parallel computing capabilities provided by GPU to achieve large-scale matrix calculation, CPU is still needed to achieve data reading and preprocessing. Therefore, when building a deep learning platform, the CPU performance should also be taken into consideration. The CPU of the deep learning platform is Intel 7700k, the GPU is Nvidia Gtx 1080, and the GPU memory capacity is $8 \mathrm{~GB}$. The key software platform components are parallel computing framework, deep learning framework, and programming language. The experiment in this study uses the CUDA 9.0 parallel computing framework and the PyTorch deep learning framework. The experiment is mainly implemented by the Python 3.6 programming language. The deep learning platform chose the Ubuntu 16.04 operating system because it has good support for deep learning-related software and hardware. Many open source frameworks are implemented based on the Ubuntu 16.04 operating system and then ported to other operating systems.

3.2. Communication Technology between the Assessment Controller and the Host Computer. The assessment controller is responsible for the transmission of instructions and status information and controls the operation of the entire pitch assessment system. It is the center of the entire system control. The main task of the assessment controller is data transmission. There are three main parts: RS232 serial port and upper computer wired communication, ZigBee and anthropometric terminal wireless communication, and RS485 serial port and LCD display terminal wired communication. This design selects the widely used RS232 serial communication method.

3.3. Communication Technology between the Assessment Controller and the Human Body Posture Measurement Terminal. The human body posture measuring terminal is installed on the human body to measure the human body's 
TABLE 1: Operating system configuration.

\begin{tabular}{lc}
\hline Operating system & Ubuntu 16.04 \\
CPU & Intel 7700k \\
GPU & Nvidia Gtx 1080 \\
Parallel computing framework & CUDA 9.0 \\
Deep learning framework & PyTorch \\
Programming language & Python 3.6 \\
\hline
\end{tabular}

movement posture. In order not to affect the actions of the reference personnel and the reliability of communication, this design uses wireless communication as the means of communication between the assessment controller and the human body posture measurement terminal. During the sit-up assessment, the distance between the two reference personnel is relatively large, the maximum is $2 \mathrm{~m}$, and the entire assessment site is up to $5 \mathrm{~m} \times 8 \mathrm{~m}$, which belongs to the short-distance category. Considering all aspects, the ZigBee wireless communication technology suitable for shortrange wireless communication is selected.

3.4. "Handshake" between Upper and Lower Computers. The lower computer receives the test data of muscle strength transmitted by the measuring device and stores it in the external memory of the single-chip microcomputer. Then, the lower computer sends a handshake request to the upper computer. If the handshake is unsuccessful, it will send the request repeatedly. If it exceeds three times, the system will prompt an error to exit. If the handshake is successful, it will start to enter the next stage, and the host computer will begin to receive test data.

3.5. Test Data Receiving and Storage. After the successful handshake between the upper and lower computer, the upper computer initializes the serial port, configures the serial port parameters, and is ready to receive data. Then, the upper computer sends signals to the lower computer and receives data through the serial port. The upper computer simultaneously checks whether the received data is complete according to the agreement between the upper and lower computers. If the received data is incomplete, it will be resent. If the received data is complete, it will undergo corresponding format conversion and store the data in the designated database.

3.6. Microcontroller Module Design. This design intends to use ZigBee wireless communication technology as the communication method of the human body posture measurement terminal and the assessment controller, and it is necessary to select a suitable wireless transceiver chip. This design uses the CC2430 chip introduced by TI. It combines an industrial-grade enhanced 8051 controller and a highperformance $2.4 \mathrm{GHz}$ ZigBee RF transceiver chip, which meets the needs of control and communication and is a small measurement terminal. It provides great convenience.

3.7. Preprocessing and Data Enhancement. The input image is subject to preprocessing steps of scaling and normalization (normalization). The image is scaled to a resolution of 224 $\times 224$ during input. This step is performed to achieve data parallel training and reduce the time consumption of neural network training. Data standardization can speed up training. In the actual implementation process, the mean and variance of each channel of RGB of the Image-Net dataset are used. The mean vector is $\mu=[0.485,0.456,0.406]$, and the variance vector is $\delta=[0.229,0.224,0.225]$.

The parameter of deep neural network is very large, and the model with high training complexity will need massive data. Data augmentation can effectively expand the data diversity of the training set through flip, translation, rotation, and other methods. Three methods of random horizontal flip, random rotation, and color change are used to change the image to achieve the effect of expanding the dataset. For each input image, determine whether to flip horizontally according to the probability of 0.5 . This data enhancement method is used because the static image data used uses different camera settings in the historical acquisition process, some of which are camera settings. The images taken by the left and right cameras are flipped horizontally. At the same time, each image is randomly rotated within the range of $[-10,10]$ according to the image center, which is in line with the characteristic that different cameras cannot guarantee the same angle during the placement process. Brightness, contrast, and saturation are randomly changed in the range of $[0.5$, 1.5] according to a uniform distribution, and the hue (hue) is randomly changed in the range of $[-0.2,0.2]$ according to a uniform distribution.

3.8. Information Management Module Design. The information management module refers to the management of some basic information that students, physical fitness test teachers, and counselors need to use during the test. This basic information is collected by the administrator before the physical fitness test and input in advance. The system, in this way, can assign the user name and password of the student, the physical test teacher, and the counselor. With the user name and password, the teacher and student can log in to the system to perform the physical test appointment operation. Whether it is a teacher or a student, their login accounts are all only one, and the input, maintenance, and update of this basic information are all operated by the system administrator.

The use of barcode scanners is mainly used for candidates who failed to take the exam due to special circumstances. When the barcode scanner scans the barcode of the candidate's admission ticket, the focus will automatically focus on the candidate's name. Recheck the candidates' names in the entire data sheet to find them. After the barcode scanner scans the barcode, the admission ticket number is automatically entered into the text box focused by the mouse, which is equivalent to manual input and pressing the enter key.

3.9. Test and Evaluation Module Design. This module is to evaluate various test indicators of healthy physical fitness. The test indicators included in the central lung fitness item include resting heart rhythm, vital capacity, 3-minute step test, systolic blood pressure, and diastolic blood pressure, and the test indicator of muscle strength is grip strength. 
And muscular endurance is measured by sit-ups within 1 minute. The flexibility of the body is measured by the degree of flexion in the sitting position. The body composition items are height, weight, BMI value, waist circumference, and hip circumference to measure.

In addition, there are extended indicators, including reaction time, standing time on one foot with closed eyes, blood lipid indicators, and blood glucose indicators. According to the measurement values of each index in different reference ranges, the evaluation can be divided into several standards: excellent, good, fair, acceptable, and poor. If it is a blood pressure index, the evaluation criteria can be normal blood pressure, normal blood pressure, mild hypertension, moderate hypertension, and severe hypertension. If it is blood lipid and blood sugar index, the evaluation criteria can be divided into low, normal, and high. If it is body weight, the evaluation criteria are underweight, normal, and overweight.

3.10. Design of Performance Management Module. The physical fitness test score management module is used by physical fitness teachers and students or counselors after the corresponding physical fitness test is over, in order to enter and query the students' scores after completing the physical fitness test. The physical fitness test teacher logs in to the system through the teacher to summarize the physical fitness and the test scores are entered into the corresponding student information in the system. After the scores are entered, the teacher can also view them at any time, as well as query the analysis of the data. The students or counselors can log in to the system on the interface to query their (the class) physical test scores.

3.11. Test Evaluation Function Module. Test purpose: check whether the link of each test item is correct and whether each test index is wrong. After the test data is entered for each index item, whether it can be correctly evaluated, whether the evaluation result is displayed in the form of graphics and text, and whether there is a comprehensive evaluation after the completion of the test of each index item. Check whether the data can be saved and printed correctly after user testing.

\section{Student Physical Fitness Test System}

4.1. Recognition Results. The accuracy rate and average accuracy rate of the designed behavior recognition model in each category are compared with the baseline model MSN. The identification analysis results are shown in Table 2. The average accuracy of MSN in the five categories is $80.31 \%$, and the behavior recognition model achieved by the dual-stream neural network and gesture recognition model in this study reached $83.2 \%$. This study did not compare its operating speed in this part, because the model implemented in this study uses the optical flow algorithm FlowNet2, which is faster than the EpicFlow used by MSN, and the dual-stream neural network is much simplified than MSN, so there must be some operating speed. As mentioned earlier, the behavior recognition model implemented in this study is better than the MSN model in terms of speed and accuracy. Because this
TABLE 2: Identify analysis results.

\begin{tabular}{lcc}
\hline $\begin{array}{l}\text { Result } \\
\text { Model }\end{array}$ & Two stream+FlowNet2 & MSN \\
\hline REACH & $93.1 \%$ & $89.74 \%$ \\
RETRACT & $94.3 \%$ & $90.47 \%$ \\
HAND IN SHELF & $68.66 \%$ & $65.56 \%$ \\
INSPECT PRODUCT & $83.9 \%$ & $82.7 \%$ \\
INSPECT SHEIF & $76 \%$ & $73.09 \%$ \\
MEAN & $83.2 \%$ & 80.31 \\
\hline
\end{tabular}

research can capture fine-grained behaviors very well and accurately, this research can achieve performance that exceeds that of complex MSN only through dual-stream neural networks.

4.2. Algorithm Performance Test Analysis. The attitude process changes smoothly, the cycle is relatively unchanged, and the peak angle is about 5 degrees to 50 degrees. The algorithm performance test result is shown in Figure 1. The overall shape of Figure 1 is similar to the analysis, but there is a big difference in the local area. There is a large jitter in the extreme part, and the cycle change is large; the peak-topeak value of the first half of Figure 1 is large, and the peak value is gradually in the second half due to physical consumption. Reduce, there is greater jitter at large angles. It can be seen from the above angle curve that the actual situation is more complicated, and the algorithm should be able to avoid any problems in the actual situation and accurately record the number of effective motion cycles. Taking situps as an example, the human body posture measurement terminal is installed on the tester's head as a training cap. The tester does sit-ups normally, and the measurement terminal performs angle capture and counting operations. Here, the standard angle is set to $90^{\circ}$. Use the serial port to output the real-time angle measured by the measuring terminal and the current count result, use the serial debugging assistant to record these two values, and then import EXCEL to draw the corresponding chart. In order to read the graph clearly and conveniently, the count value is multiplied by 10. The ordinate corresponding to the horizontal line in Figure 1 is 10 times the count value. It can be seen that the human body posture measurement terminal has performed the correct count. The initial angle of the tester when lying down is $90^{\circ}$, and the angle when sitting up is $0^{\circ}$, at the moment of lying down, the tester did 10 sit-ups, but the seventh one was not standard and did not reach $90^{\circ}$, the measuring terminal did not count, and the final count was 9.

4.3. Muscle Strength Test Analysis. Use different colors to show the strength curves of the four tested individuals on different graphs. The four tested individuals can choose any specified conditions. The color of the curve can be changed. Click any graph to display its detailed information. The muscle strength test results are shown in Figure 2. It can be seen from Figure 2 that the same figure is more obvious, and it can be seen that the grip strength curves caused by the differences between the two test individuals are significantly different. 


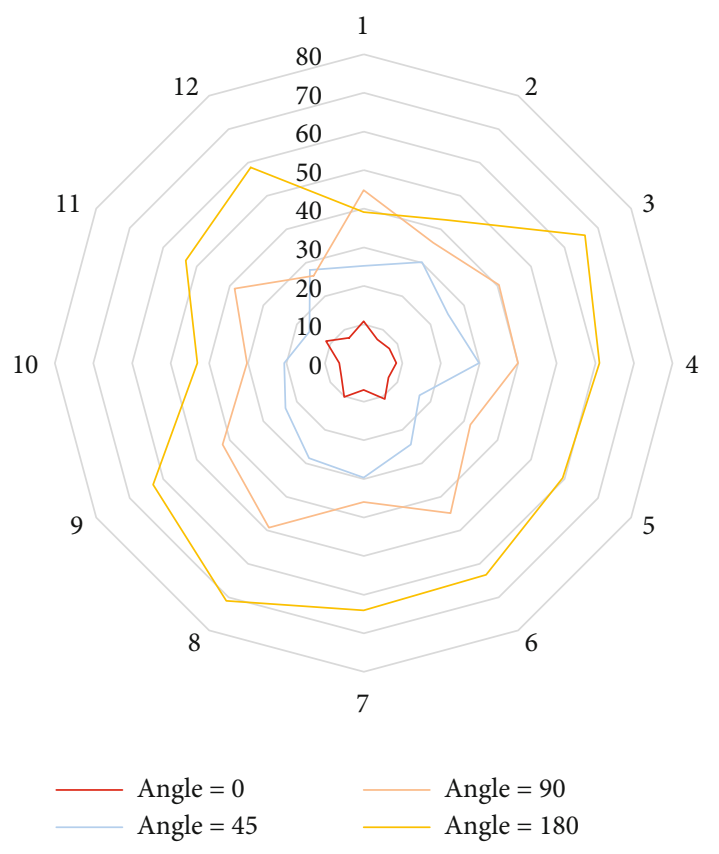

Figure 1: Algorithm performance test results.

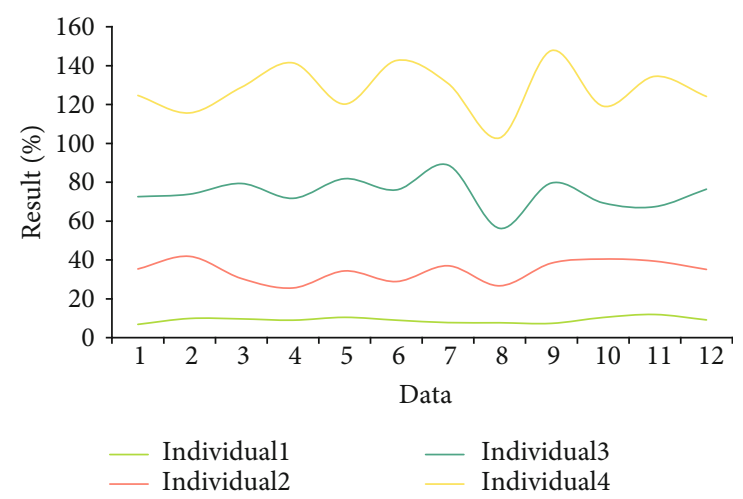

FIGURE 2: Muscle strength test results.

The dynamic data model of this sample set is obtained through statistical calculation steps on the data of 35 individuals participating in the test using statistical principles. Because the selected experimenters are all students of the same age, there is no need to redivide the two fields of age and work nature. First, use the system function to select gender: male, test item: right grip strength, search in the database and find that meets the conditions, and then, count the searched data, store the data obtained from the test data table into an array, and then, take the next testee's data and add them to the data group in the corresponding order of time until all matches conditional data are all superimposed, and finally, each number in the array is averaged to get a dynamic data model. It can be seen that under the same time setting, the man's curve is short and the peak is prominent, the woman's curve is long and the trend is relaxed, and the man is in the comparison of the absolute strength, explosive power, rapid reaction coefficient, and other human parameters, having an advantage over women, and women have an advantage over men in endurance.
4.4. System Performance Test. In order to facilitate user operation and achieve good human-computer interaction, the interface design of the system adopts an all-Chinese graphic frame structure, with friendly interface design and simple operation. The interface item side is the traditional dropdown menu and toolbar; the left side of the interface adopts the popular tree-like menu structure, and clicking can produce the same effect as the drop-down menu, making the human-computer interaction faster and more practical, and the interface is more beautiful; the right side is the interface. The main display area can display graphics, data, tables, etc. The system performance test result is shown in Figure 3. All functions can be automatically completed by clicking the menu or button with the mouse. The main menu of the system includes data acquisition, data query, graph display, data analysis, comparison, and data output. This research is based on the theory of serial communication between the computer and the single-chip computer and makes full use of the powerful data storage and processing ability of the $\mathrm{PC}$ and the communication technology, as well as the data acquisition, keyboard input, and display functions of the single-chip computer to develop a basketball special comprehensive test system. The system has been debugged and developed, and various functions have been basically realized and have been used in physical fitness tests. Through continuous debugging and trial and error in the process of system development and development and with the changes in sports college entrance examination requirements, the function is further improved. System is reasonable in design, advanced in technology, accurate in data transmission, data processing, and score recording, and has reached expectations. The effect also played a powerful role in practical applications, which greatly improved the efficiency and accuracy of sports testing.

The student physical test data analysis system can change that the students taking the test are no longer passively tested, but can choose the appropriate test time and subjects according to their own personal situation. At the same time, for physical fitness test teachers, after the on-site test is completed and recorded, there is no need to pass it layer by layer. It is first handed over to the Sports Basic Department, then distributed to the departments, and finally transmitted to the students by the instructor. It is also prone to errors. The data analysis result of the student's physical fitness test is shown in Figure 4. The system can directly enter the scores by the physical fitness test teacher. After the entry is completed, the students can log in and view by themselves. It is also convenient for students to compare their physical fitness test with other students, changing the phenomenon that students only care about whether their physical fitness test scores have passed. The system greatly improves the accuracy and timeliness of data, reducing the workload of the Sports Basic Department and the instructors and department staff. It has a huge positive effect on the management of students' physical fitness test. It receives a segment as input, and the time series flow part of the model receives motion information. A segment is composed of 6 frames of images, and two successive images calculate 5 sets of optical flow results from each other, that is, 10 frames of optical flow information 


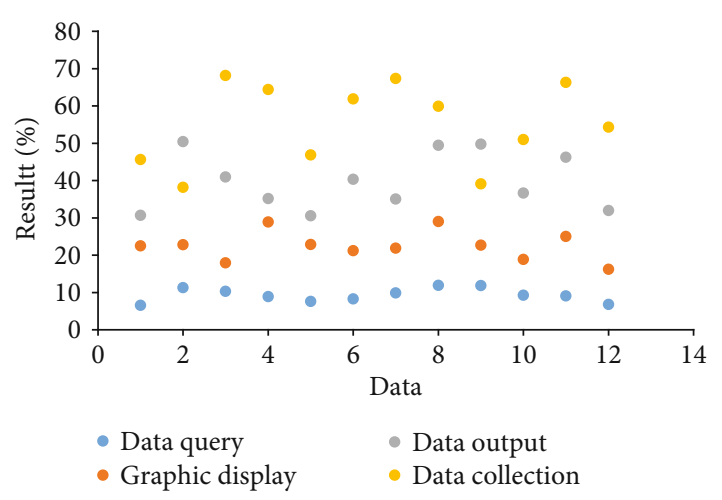

FIGURE 3: System performance test results.

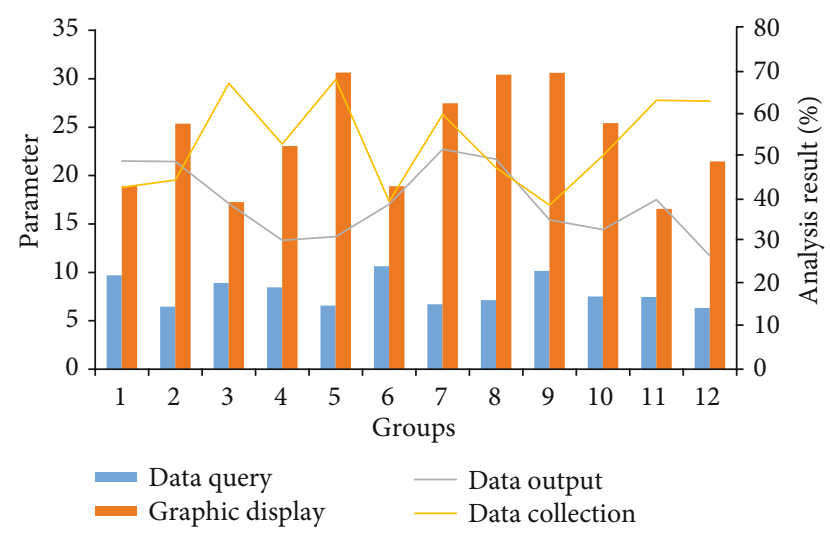

Figure 4: Data analysis results of student physical fitness test.

( $x$ direction and $y$ direction). Correspondingly, the timing stream receives 10 channels of data. In order to change the original VGG network from receiving 3 channels of RGB images to receiving 10 channels of optical flow information, the author calculated the input layer along the dimension of the input channel, the weight of the convolution kernel, and copy the weight average to 10 channels. The size of the optical flow data is $256 \times 256$ dimensions, and the $8 \times 8 \times$ 512 dimension feature map is obtained after the calculation of the convolutional layer. Immediately after that, there are two fully connected layers with dimensions of $32768 \times 4096$ and $4096 \times 5$. The spatial stream receives an ordinary RGB image, and it randomly selects a frame of image from the input segment as input. After the calculation of the convolutional layer, it obtains a feature map of $7 \times 7 \times 512$ dimensions. Similarly, it connects two layers of fully connected layers. The dimensions are $25088 \times 4096$ and $4096 \times 5$.

\section{Conclusion}

The parameter of deep neural network is very large, and the model with high training complexity will need massive data. Data augmentation can effectively expand the data diversity of the training set through flip, translation, rotation, and other methods. Three methods of random horizontal flip, random rotation, and color change are used to change the image to achieve the effect of expanding the dataset. For each input image, it is determined whether to flip horizontally according to a certain probability. This data enhancement method is used because the static image data used uses different camera settings in the historical acquisition process, some of which are camera settings. The images taken by the left and right cameras are flipped horizontally.

The physical fitness test score management module is used by physical fitness teachers and students or counselors after the corresponding physical fitness test is over, in order to enter and query the students' scores after completing the physical fitness test. The physical fitness test teacher logs in to the system through the teacher to summarize the physical fitness and the test scores are entered into the corresponding student information in the system. After the scores are entered, the teacher can also view them at any time, as well as query the analysis of the data. The students or counselors can log in to the system on the interface to query their (the class) physical test scores.

This research mainly discusses the design of student physical test system and test data analysis system based on computer vision. This research is mainly based on the motion recognition algorithm for motion recognition. In the hardware configuration, the key is CPU and GPU. The model realizes large-scale matrix calculation based on the parallel computing capability provided by GPU and uses $\mathrm{CPU}$ to realize data reading and preprocessing. The assessment controller is responsible for the transmission of instructions and status information and controls the operation of the entire pitch assessment system. It is the center of the entire system control. ZigBee wireless communication technology is used as the communication method between the human body posture measurement terminal and the assessment controller. The input image is subject to preprocessing steps of scaling and standardization. Three methods of random horizontal flip, random rotation, and color change are used to change the image to achieve the effect of expanding the dataset, and then, the test evaluation module is used to evaluate various test indicators of the body.

\section{Data Availability}

The data that support the findings of this study are available from the corresponding author upon reasonable request.

\section{Conflicts of Interest}

There are no potential competing interests in our paper. And all authors have seen the manuscript and approved to submit to your journal.

\section{Acknowledgments}

This work was supported by Key Topics of the Higher Education Academy of China-research on the historical evolution and realistic enlightenment of the development of high-level sports teams in universities in China (17TZ010). 


\section{References}

[1] I. Kitouni, D. Benmerzoug, and F. Lezzar, "Smart agricultural enterprise system based on integration of Internet of things and agent technology," Journal of Organizational and End User Computing, vol. 30, no. 4, pp. 64-82, 2018.

[2] Z. Lv, L. Qiao, D. Chen, R. Lou, J. Li, and Y. Li, "Machine learning for proactive defense for critical infrastructure systems," IEEE Communications Magazine, 2020.

[3] B. T. Grys, D. S. Lo, N. Sahin et al., "Machine learning and computer vision approaches for phenotypic profiling," Journal of Cell Biology, vol. 216, no. 1, pp. 65-71, 2016.

[4] S. Ding, S. Qu, Y. Xi, and S. Wan, "A long video caption generation algorithm for big video data retrieval," Future Generation Computer Systems, vol. 93, pp. 583-595, 2019.

[5] S. Yeung, N. L. Downing, L. Fei-Fei, and A. Milstein, "Bedside computer vision-moving artificial intelligence from driver assistance to patient safety," New England Journal of Medicine, vol. 378, no. 14, pp. 1271-1273, 2018.

[6] Y. J. Chang and Y. S. Ho, "Pixel based cost computation using weighted distance information for cross-scale stereo matching," Electronic Imaging, vol. 2016, no. 10, pp. 1-6, 2016.

[7] M. H. Zaki, T. Sayed, and X. Wang, "Computer vision approach for the classification of bike type (motorized versus non-motorized) during busy traffic in the city of Shanghai," Journal of Advanced Transportation, vol. 50, no. 3, 362 pages, 2016.

[8] A. B. Meireles, A. W. Vieira, L. Corpas et al., "Dental wear estimation using a digital intra-oral optical scanner and an automated 3D computer vision method," Computer Methods in Biomechanics \& Biomedical Engineering, vol. 19, no. 5-8, pp. 507-514, 2015.

[9] L. Wang, Y. Zhao, Y. Zhou, and J. Hao, "Calculation of flexible printed circuit boards (FPC) global and local defect detection based on computer vision," Circuit World, vol. 42, no. 2, pp. 49-54, 2016.

[10] S. Wan, Z. Gu, and Q. Ni, "Cognitive computing and wireless communications on the edge for healthcare service robots," Computer Communications, vol. 149, pp. 99-106, 2017.

[11] A. Barbu, Y. She, L. Ding, and G. Gramajo, "Feature selection with annealing for computer vision and big data learning," IEEE Transactions on Pattern Analysis \& Machine Intelligence, vol. 39, no. 2, pp. 272-286, 2017.

[12] J. Wäldchen and P. Mäder, "Plant species identification using computer vision techniques: a systematic literature review," Archives of Computational Methods in Engineering, vol. 25, no. 2, pp. 507-543, 2018.

[13] Y. Jiang, H. Song, R. Wang, M. Gu, J. Sun, and L. Sha, "Datacentered runtime verification of wireless medical cyberphysical system," IEEE Transactions on Industrial Informatics, vol. 13, no. 4, pp. 1900-1909, 2017.

[14] A. K. Patel and S. Chatterjee, "Computer vision-based limestone rock-type classification using probabilistic neural network," Geoscience Frontiers, vol. 7, no. 1, pp. 53-60, 2016.

[15] M. Nagle, K. Intani, G. Romano, B. Mahayothee, V. Sardsud, and J. Müller, "Determination of surface color of 'all yellow' mango cultivars using computer vision," International Journal of Agricultural and Biological Engineering, vol. 9, no. 1, pp. 4250, 2016.

[16] J. Braz, J. Pettré, and P. Richard, "Computer vision, imaging and computer graphics. Theory and applications," Communi- cations in Computer \& Information ence, vol. 229, no. 25, pp. 837-837, 2016.

[17] L. Lopez-Fuentes, V. D. W. Joost, M. González-Hidalgo et al., "Review on computer vision techniques in emergency situation," Multimedia Tools \& Applications, vol. 77, no. 13, pp. 1-39, 2017.

[18] T. Hoyoux, A. J. Rodriguez-Sanchez, and J. H. Piater, "Can computer vision problems benefit from structured hierarchical classification?," Machine Vision \& Applications, vol. 27, no. 8, pp. 1299-1312, 2016.

[19] M. I. Rathore, "Computer vision syndrome-an emerging occupational hazard," Research journal of science and technolo, vol. 9, no. 2, pp. 293-297, 2017.

[20] S. Khan, H. Rahmani, S. A. A. Shah, and M. Bennamoun, "A guide to convolutional neural networks for computer vision," Synthesis Lectures on Computer Vision, vol. 8, no. 1, pp. 1207, 2018.

[21] Z. Lv and A. K. Singh, "Big data analysis of Internet of things system," ACM Transactions on Internet Technology (TOIT), 2020.

[22] S. Y. Du and Z. G. Liu, "A comparative study of different color spaces in computer-vision-based flame detection," Multimedia Tools and Applications, vol. 75, no. 17, pp. 10291-10310, 2016.

[23] J. Malik, "Technical perspective: what led computer vision to deep learning?," Communications of the ACM, vol. 60, no. 6, pp. 82-83, 2017.

[24] O. Zendel, M. Murschitz, M. Humenberger, and W. Herzner, "How good is my test data? Introducing safety analysis for computer vision," International Journal of Computer Vision, vol. 125, no. 1-3, pp. 95-109, 2017.

[25] T. Charles, "Photogrammetric computer vision: statistics, geometry, orientation and reconstruction," Photogrammetric Engineering \& Remote Sensing, vol. 83, no. 10, pp. 661-662, 2017.

[26] L. Lopez-Fuentes, J. van de Weijer, M. González-Hidalgo, H. Skinnemoen, and A. D. Bagdanov, "Review on computer vision techniques in emergency situation," Multimedia Tools \& Applications, vol. 77, no. 13, pp. 1-39, 2017. 